\title{
Lung transplantation in elderly patients
}

\author{
Andrew Courtwright ${ }^{1}$, Edward Cantu ${ }^{2}$ \\ ${ }^{1}$ Division of Pulmonary and Critical Care Medicine, ${ }^{2}$ Division of Cardiovascular Surgery, University of Pennsylvania School of Medicine, \\ Philadelphia, PA, USA \\ Contributions: (I) Conception and design: All authors; (II) Administrative support: None; (III) Provision of study materials or patients: None; \\ (IV) Collection and assembly of data: All authors; (V) Data analysis and interpretation: All authors; (VI) Manuscript writing: All authors; (VII) Final \\ approval of manuscript: All authors. \\ Correspondence to: Edward Cantu, MD, MSCE. Associate Professor of Surgery, Division of Cardiovascular Surgery, University of Pennsylvania School \\ of Medicine, 3400 Spruce Street, 6 Silverstein Pavilion, Philadelphia, PA 19104, USA. Email: Edward.Cantu@uphs.upenn.edu.
}

\begin{abstract}
Consensus statements on the selection of lung transplant candidates have consistently identified older age as a relative contraindication to transplantation. A combination of population-level demographic changes, revision of the lung allocation score (LAS), and clearer data on outcomes in elderly transplant recipients has, however, driven a steady increase in the threshold at which age is taken into consideration. This article reviews the current state of lung transplantation in elderly patients with an emphasis on the factors that have increased lung transplantation in older age groups, their expected outcomes including survival and health-related quality of life, and the factors that go in to appropriate candidate and procedure selection in this population.
\end{abstract}

Keywords: Age; elderly; lung allocation; lung transplantation

Submitted Apr 20, 2017. Accepted for publication Jul 26, 2017.

doi: 10.21037/jtd.2017.08.31

View this article at: http://dx.doi.org/10.21037/jtd.2017.08.31

\section{Introduction}

Consensus statements on the selection of lung transplant candidates have consistently identified older age as a relative contraindication to transplantation. International guidelines in the 1990s recommended against bilateral lung transplant in individuals over 60 and against single transplant in those over 65 (1). By the mid-2000s, the guidelines were revised to state that, although there is no upper age limit representing an absolute contraindication to transplantation, age above 65 should be considered a relative contraindication (2). The most recent guidelines again characterize age over 65 as a relative contraindication but explicitly identify age greater than 75 as the threshold at which individuals are unlikely to be candidates (3). A combination of population-level demographic changes, revision of the lung allocation score (LAS), and clearer data on outcomes in elderly transplant recipients has driven the steady increase in the threshold at which age is taken into consideration and the strength of that recommendation. In this article, we review the current state of lung transplantation in elderly patients.

\section{Factors driving increased transplantation in older patients}

In the United States (US), the portion of lung transplant recipients over age 65 has grown $430 \%$ over the last 13 years from $6.9 \%$ in 2004 to $29.6 \%$ in 2016 (Figure 1). Internationally, recipients over age 65 increased from $2.6 \%$ to almost $17 \%$ over a similar time. In both populations, the proportion of transplant recipients over 70 increased from less than half a percent to almost 3\% by mid-2012. Demographic, clinical, and lung allocation-related reasons accounted for these changes.

\section{Aging population}

With the exception of cystic fibrosis (CF) and certain forms of pulmonary arterial hypertension, the incidence and prevalence of end-stage lung diseases increase with age. 


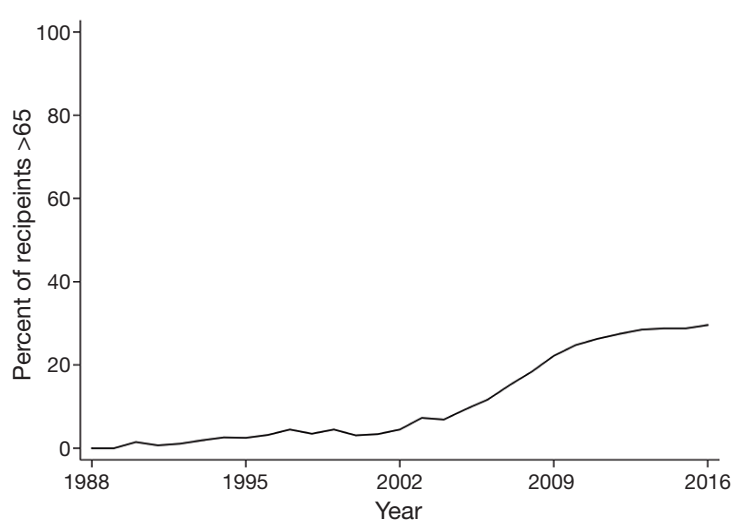

Figure 1 Trends in lung transplantation in the US among recipients $>65$.

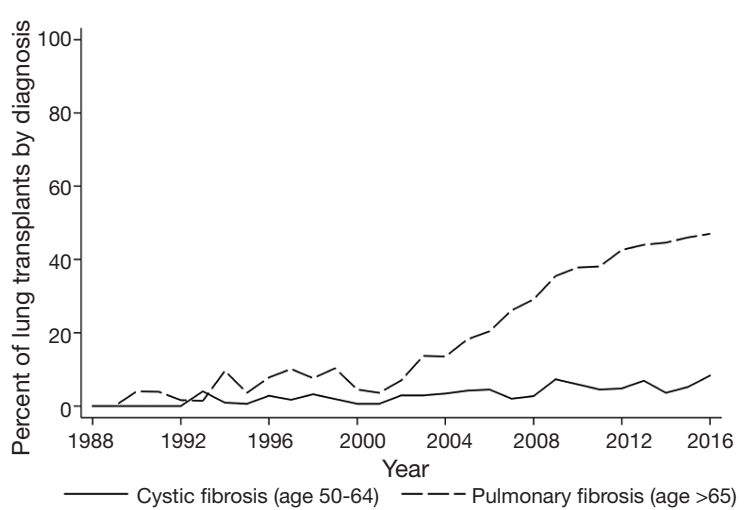

Figure 2 Trends in lung transplantation in the US among older age groups in cystic fibrosis and pulmonary fibrosis.

For example, idiopathic pulmonary fibrosis (IPF), one of the leading indications for transplantation, is significantly more common after age 60 and appears to have the highest prevalence among individuals $>70(4,5)$. While the epidemiology of IPF varies by geographic region, most studies have reported an increased disease prevalence over the last twenty years, particularly in individuals $>65$ (6). Concordant with this change, the percentage of IPF transplants performed in patients over age 65 in the US has risen from $7.8 \%$ in 1996 to $20.4 \%$ in 2006 to $40.7 \%$ in 2016 (Figure 2). Single center studies have also shown an increase in the percent of patients with IPF referred for transplantation evaluation, from $19.8 \%$ in the early 1990 s to $30.8 \%$ in the mid-2000s $(7,8)$. Larger database studies are necessary to confirm referral trends, although the overall increase in transplants performed for patients with IPF is at least, in part, a function of the rising prevalence of the disease in an aging population.

\section{Treatment for younger patients}

At the same time, advances in the care of patients with genetic lung diseases, particularly CF, has increased survival and delayed the need for lung transplantation, when necessary, until older age (9). Although we are unaware of any published data on the average age of referral for lung transplant in CF, the fraction of lung transplants performed among CF patients above 50 has steadily increased from $2.8 \%$ in 1996 to $4.5 \%$ in 2006 to $8.3 \%$ in 2016 (Figure 2). Similarly, the proportion of CF transplants for patients $>65$, while still extremely small, has steadily increased over the past 20 years. The advent of targeted therapy to improve CFTR function, thereby slowing the decline in lung function, is anticipated to delay the need for transplantation in the CF population, thereby further increasing age at referral (10).

\section{LAS changes}

Although the increase in median age of lung transplant recipients began in the late 1990s, the 2005 revision of the LAS accelerated this trend in the US. Prior to that year lungs were allocated based on duration of waitlist time after matching for size and ABO blood type. In May 2005, the LAS was changed to emphasize medical need rather than wait time. The new scoring system weighed anticipated 1 -year survival without transplant-so called wait list urgency-twice as heavily as predicted 1-year survival following transplant. Age was included as a variable that impacted the calculation of both waitlist and post-transplant survival although age and LAS score are not related in a linear manner. More importantly than the inclusion of age itself, the LAS gave increased priority to diseases with higher short-term mortality such as IPF that are also more common in older patients. As a consequence, the LAS shifted transplant toward older, sicker recipients (11).

\section{Program willingness}

As revisions in allocation schemes and clinical and demographic changes have driven lung transplantation toward older patients, centers have gained greater familiarity with transplantation in this population. In general, however, elderly transplant recipients tend to cluster in high volume programs, suggesting that there is 
some combination of willingness and experience with this population that has driven increased transplantation. For example, in the LAS era, recipients $>70$ are transplanted in centers with an average volume of 34 transplants per year and the increase in lung transplantation in patients $>70$ appear to have primarily occurred in centers that perform more than 30 transplant per year $(12,13)$. It is unclear to what extent "specialized" experience in the care of elderly recipients has allowed these centers to increase transplants in this population and the extent to which smaller volume centers could expect similar outcomes.

\section{Outcomes in older patients}

\section{Perioperative morbidity and mortality}

Despite concerns that elderly patients may have reduced functional reserve, placing them for increased risk for perioperative mortality; several large studies have shown no difference in in-hospital or 30-day mortality for older recipients (14). For example, Biswas Roy et al. found a $96 \%$ survival at 30 days in single lung transplant recipients $<65$ compared to $96 \%$ for those $65-69,97 \%$ for those $70-74$, and $96 \%$ for those $75-79$. Bilateral recipients had similar age-related 30-day survival with the exception of those $75-79$ where survival was only $73 \%$ (15). An analysis of the US Medicare claims database suggests no difference in in-hospital, 30 or 90 -day mortality for recipients $>70$ compared to those 60-69 and those $<60$ (13). In this cohort and others, recipients $>70$ have similar length of hospital stay to those 60-69 and shorter length of stay compared to those $<60$, possibly reflecting increased use of single lung transplant in this population $(13,14)$. Older recipients, however, have higher rates of 30- and 90-day readmission and age is an independent risk factor for discharge to an inpatient rehabilitation facility following transplant $(13,16)$.

\section{Long-term survival and cause of death}

Several studies of long-term survival before the LAS era found reduced long-term survival for older transplant recipients. For example, Gutierrez et al. reported a 37\% vs. $57 \%$ adjusted 5 -year survival for recipients $>60 v s$. those below 60. Similarly, larger cohort studies of US lung transplants before 2005 demonstrated that age $>70$ was a significant, independent risk factor for 1- and 3-year mortality $(14,17)$. Studies in the post-LAS era, however, have found conflicting results in survival differences between patients 60-69 years old and $>70$ with some reporting equivalent 1-year survival and others showing slightly worse 1 - and 3 -year survival, depending on the population $(13,14)$. There are consistent data, however, that recipients over 65 have worse mortality than those below 65 . For example, the 3 -year survival rate for single lung transplant in 12-64 vs. 65-69 vs. $70-74$ year olds drops from $65 \%$ to $57 \%$ to $49 \%$ and from $68 \%$ to $63 \%$ to $57 \%$ for bilateral transplants. Finally, despite case reports of successful transplantation in octogenarians, large cohort studies have consistently shown poor intermediate and long-term outcomes for recipients over age 75 (18). A recent study of survival in recipients over 75 demonstrated 1 - and 3-year survival of $51 \%$ and $26 \%$, respectively. In the same study, 5-year survival could not be calculated because of the small number of long-term survivors (15).

Despite early concerns that older recipients may be more susceptible to severe infections because of immunosenescence, recent large cohort studies have demonstrated no difference in the incidence of fatal infection in recipients $>65$ and $>70$ compared to younger recipients $(15,17,18)$. This may be a function of protocols designed to reduce induction and maintenance immunosuppression in these groups (19). Rates of fatal malignancies and vascular events appear to be more common in patients $>65$ and $>70(14,16,17)$. Graft failure as a cause of death, however, declines significantly in patients $>70$ (14). Single center studies have also suggested that older recipients are at higher risk for drug toxicity, non-fatal malignancies (particularly skin cancer), and cognitive decline suggesting increased need for drug monitoring and dermatologic surveillance in this population $(20,21)$.

\section{Health related quality of life (HRQL)}

Given their increased morbidity following transplant, it might be expected that the HRQL benefit would be less for older patients. Cohort studies, however, have conflicted regarding the impact of age on the survival-adjusted HRQL benefit of transplant. Singer et al. found, in a single center prospective study, that the significant improvements in multiple HRQL assessments following transplant were not meaningfully different by recipient age, when treated as a continuous variable (22). Only $14 \%$ of their cohort (46 patients) were over age 65 , however, and only $2 \%$ (8 patients) were over age 70 and they did not specifically examine HRQL in these populations compared to younger recipients. In a separate cohort study, researchers found 
that, although patients over 65 did have increases in HRQL scores following transplant, they had substantially smaller changes compared to younger patients (23). Finally, in a large cohort study of US transplants from 2005-2009, there was no difference in rate of functional decline, as measured by Karnofsky performance score, in recipients 65-69 and those $>70$ compared to younger recipients. Recipients older than 70, however, had worse overall post-transplant functional status than those 65-69, who, in turn, had worse functional status than those $<65(13,24)$.

\section{Appropriate candidate and procedure selection}

In order to maintain equivalent or near-equivalent morbidity and short-term mortality for elderly $v s$. nonelderly recipients, careful candidate, donor, and procedure (single vs. bilateral transplant) selection is paramount. Chief among these considerations is differentiating chronologic age from functional status and the accumulation of agingrelated comorbidities.

\section{Candidate selection}

In the largest cohort studies demonstrating similar perioperative outcomes for older $(>65$ and $>70)$ recipients, older patients had notably few co-morbidities and were relatively healthy at the time of transplant. For example, Hayanga et al. found that almost no recipients above 70 had an abnormal creatinine or total bilirubin; that only $16.8 \%$ had diabetes; that only $13.4 \%$ were hospitalized at the time of transplant (6.1\% in the ICU); and that only $1.2 \%$ required intra-operative extracorporeal membrane oxygenation (ECMO) support (14). Similarly, Biswas Roy et al. reported that, among transplant recipients $>75$, only $1 \%$ were ventilated pre-transplant and that mean pulmonary arterial pressure was significant lower [22.6 vs. 27.5 millimeters mercury $(\mathrm{mmHg})]$ compared to recipients <65 (15). Despite case reports of successful use of ECMO as a bridge to transplantation in patients above 70 years, there are no data to support the routine use of pre-transplant mechanical ventilation or circulatory support in this population (25).

Although there are limited comparative data on frailty measures in elderly $v s$. non-elderly recipients, given the association between frailty and increased post-transplant mortality, it is likely that centers also consider objective and subjective measures of frailty when accepting elderly candidates for listing (26). Similarly, there are no data on transplantation in elderly patients with vascular co- morbidities such as coronary artery disease, carotid disease, or peripheral vascular disease or other age-related diseases such as swallowing dysfunction or neurocognitive impairment. In general, however, ideal elderly candidates should be free from other organ system dysfunction that might negatively impact their post-transplant course.

\section{Donor selection}

Given the worse long-term survival among elderly transplant candidates, there has been some consideration of using lungs from extended criteria donors where reduced baseline pulmonary function may be less relevant compared to a younger candidate with longer life expectancy. For example, several single center studies have found that older recipients are more likely to undergo transplantation with lungs from donors above age 55, the typical age cutoff for considering a donor acceptable rather than ideal $(20,27,28)$. In large cohort studies, the use of lungs from donors $>55$ does not appear to adversely impact overall or conditional one year survival in recipients $>65$ (29). There are little data, however, on the use of donors with multiple marginal criteria such as older age and significant smoking history. Cohort studies that have demonstrated acceptable outcomes for older recipients have consistently reported a low rate of utilization of lungs from donors with any smoking history and the use of donors with $>20$ pack-year smoking has been associated with increased mortality in recipients $>60(14,30)$. Finally, single center data suggest that older recipients are less like to undergo cytomegalovirus (CMV) mismatch (donor positive, recipient negative) transplants, particularly as this has been identified as a significant risk factor for death in the elderly lung transplant population (21).

\section{Procedure selection}

The choice of whether to perform single or bilateral transplant in older recipients is an area of ongoing controversy. Although bilateral transplant conveys a significant overall survival benefit for most end-stage lung diseases, association with increased early mortality compared to single lung transplant led to early recommendations to prefer single lung for recipients $>65$ or even $>60(1,31,32)$. Advances in surgical technique, ICU and post-operative care, and immunosuppression, however, began to improve survival in elderly recipients and subsequent studies in the post-LAS era have shown increased willingness to perform bilateral transplants in these patients, including those $>70$ 
$(12,20,33)$. In a recent study of US transplants in the postLAS era, Biswas Roy et al. found no difference in 30- or 90-day survival in recipients $65-69$ or 70-74 with single $v$ s. bilateral transplant (15). They did, however, identify a trend to increased overall survival with bilateral transplant in these age groups, independent of underlying obstructive or restrictive lung disease. There was no such benefit apparent in patients above 75 . Based on these data, it may be reasonable to consider bilateral transplant in patients $<74$, assuming no other technical or acuity of illness reasons to prefer single lung transplant. Even with bilateral transplant, however, it is important to note that overall survival remains worse in patients $>70$ compared to younger recipients $(13,15)$.

\section{Resource allocation considerations}

\section{Duty to rescue and fair innings}

In weighing predicted mortality without transplant-wait list urgency-more heavily than predicted one year survival following transplant, the revised LAS prioritizes a duty to rescue over maximizing post-transplant survival benefit (34). Coupled with the changing demographics of end-stage lung disease, this has led to increased allocation to older patients, who, at the extreme, derive less long-term survival benefit from transplantation. Surveys of community beliefs about appropriate organ allocation have consistently identified prioritizing younger age-and the chance to live a normal life span or to have a fair number of life "innings" - as important to equitable allocation (35). As with current lung transplantation guidelines, respondents in these community-based surveys do not identify a specific age cutoff, but there is an ongoing tension between a commitment to fair innings and to a system that increases the number of elderly transplant recipients. While this debate continues, an ongoing commitment to careful candidate selection and post-transplant care targeted to the specific needs of this population can help to continue to improve their outcomes.

\section{Acknowledgements}

None.

\section{Footnote}

Conflicts of Interest: The authors have no conflicts of interest to declare.

\section{References}

1. Maurer JR, Frost AE, Estenne M, et al. International guidelines for the selection of lung transplant candidates. The International Society for Heart and Lung Transplantation, the American Thoracic Society, the American Society of Transplant Physicians, the European Respiratory Society. Transplantation 1998;66:951-6.

2. Orens JB, Estenne M, Arcasoy S, et al. International guidelines for the selection of lung transplant candidates: 2006 update--a consensus report from the Pulmonary Scientific Council of the International Society for Heart and Lung Transplantation. J Heart Lung Transplant 2006;25:745-55.

3. Weill D, Benden C, Corris PA, et al. A consensus document for the selection of lung transplant candidates: 2014--an update from the Pulmonary Transplantation Council of the International Society for Heart and Lung Transplantation. J Heart Lung Transplant 2015;34:1-15.

4. Nalysnyk L, Cid-Ruzafa J, Rotella P, et al. Incidence and prevalence of idiopathic pulmonary fibrosis: review of the literature. Eur Respir Rev 2012;21:355-61.

5. Collard HR, Ward AJ, Lanes S, et al. Burden of illness in idiopathic pulmonary fibrosis. J Med Econ 2012;15:829-35.

6. Raghu G, Chen SY, Yeh WS, et al. Idiopathic pulmonary fibrosis in US Medicare beneficiaries aged 65 years and older: incidence, prevalence, and survival, 2001-11. Lancet Respir Med 2014;2:566-72.

7. Egan TM, Trulock EP, Boychuk J, et al. Analysis of referrals for lung transplantation. The Washington University Lung Transplantation Group. Chest 1991;99:867-70.

8. de Pablo A, Juarros L, Jodra S, et al. Analysis of patients referred to a lung transplantation unit. Transplant Proc 2013;45:2351-6.

9. MacKenzie T, Gifford AH, Sabadosa KA, et al. Longevity of patients with cystic fibrosis in 2000 to 2010 and beyond: survival analysis of the Cystic Fibrosis Foundation patient registry. Ann Intern Med 2014;161:233-41.

10. Polenakovik HM, Sanville B. The use of ivacaftor in an adult with severe lung disease due to cystic fibrosis $(\Delta \mathrm{F} 508 /$ G551D). J Cyst Fibros 2013;12:530-1.

11. Kozower BD, Meyers BF, Smith MA, et al. The impact of the lung allocation score on short-term transplantation outcomes: a multicenter study. J Thorac Cardiovasc Surg 2008;135:166-71.

12. Kilic A, Merlo CA, Conte JV, et al. Lung transplantation in patients 70 years old or older: have outcomes changed 
after implementation of the lung allocation score? J

Thorac Cardiovasc Surg 2012;144:1133-8.

13. McCarthy F, Savino D, Graves D, et al. Cost and Readmission of Single and Double Lung Transplantation in the U.S. Medicare Population. J Heart Lung Transplant 2017;36:S115.

14. Hayanga AJ, Aboagye JK, Hayanga HE, et al. Contemporary analysis of early outcomes after lung transplantation in the elderly using a national registry. J Heart Lung Transplant 2015;34:182-8.

15. Biswas Roy S, Alarcon D, Walia R, et al. Is There an Age Limit to Lung Transplantation? Ann Thorac Surg 2015;100:443-51.

16. Tang M, Mawji N, Chung S, et al. Factors affecting discharge destination following lung transplantation. Clin Transplant 2015;29:581-7.

17. Gutierrez C, Al-Faifi S, Chaparro C, et al. The effect of recipient's age on lung transplant outcome. Am J Transplant 2007;7:1271-7.

18. Shigemura N, Brann S, Wasson S, et al. Successful lung transplantation in an octogenarian. J Thorac Cardiovasc Surg 2010;139:e47-8.

19. Mahidhara R, Bastani S, Ross DJ, et al. Lung transplantation in older patients? J Thorac Cardiovasc Surg 2008;135:412-20.

20. Vadnerkar A, Toyoda Y, Crespo M, et al. Age-specific complications among lung transplant recipients 60 years and older. J Heart Lung Transplant 2011;30:273-81.

21. Cohen DG, Christie JD, Anderson BJ, et al. Cognitive function, mental health, and health-related quality of life after lung transplantation. Ann Am Thorac Soc 2014;11:522-30.

22. Singer LG, Chowdhury NA, Faughnan ME, et al. Effects of Recipient Age and Diagnosis on Health-related Qualityof-Life Benefit of Lung Transplantation. Am J Respir Crit Care Med 2015;192:965-73.

23. Singer JP, Katz PP, Soong A, et al. Effect of Lung Transplantation on Health-Related Quality of Life in the Era of the Lung Allocation Score: A U.S. Prospective Cohort Study. Am J Transplant 2017;17:1334-45.

24. Genao L, Whitson HE, Zaas D, et al. Functional status

Cite this article as: Courtwright A, Cantu E. Lung transplantation in elderly patients. J Thorac Dis 2017;9(9):33463351. doi: 10.21037/jtd.2017.08.31 after lung transplantation in older adults in the postallocation score era. Am J Transplant 2013;13:157-66.

25. Hayanga JA, Murphy E, Girgis RE, et al. Extracorporeal Membrane Oxygenation as a Bridge to Lung Transplantation in Patients Over Age 70 Years: A Case Report. Transplant Proc 2017;49:218-20.

26. Wilson ME, Vakil AP, Kandel P, et al. Pretransplant frailty is associated with decreased survival after lung transplantation. J Heart Lung Transplant 2016;35:173-8

27. Sommer W, Ius F, Salman J, et al. Survival and spirometry outcomes after lung transplantation from donors aged 70 years and older. J Heart Lung Transplant 2015;34:1325-33.

28. Shigemura N, Horai T, Bhama JK, et al. Lung transplantation with lungs from older donors: recipient and surgical factors affect outcomes. Transplantation 2014;98:903-8.

29. Hayes D Jr, Black SM, Tobias JD, et al. Influence of donor and recipient age in lung transplantation. J Heart Lung Transplant 2015;34:43-9.

30. Nwakanma LU, Simpkins CE, Williams JA, et al. Impact of bilateral versus single lung transplantation on survival in recipients 60 years of age and older: analysis of United Network for Organ Sharing database. J Thorac Cardiovasc Surg 2007;133:541-7.

31. Fischer S, Meyer K, Tessmann R, et al. Outcome following single vs bilateral lung transplantation in recipients 60 years of age and older. Transplant Proc 2005;37:1369-70.

32. Meyer DM, Bennett LE, Novick RJ, et al. Single vs bilateral, sequential lung transplantation for end-stage emphysema: influence of recipient age on survival and secondary end-points. J Heart Lung Transplant 2001;20:935-41.

33. Palmer SM, Davis RD, Simsir SA, et al. Successful bilateral lung transplant outcomes in recipients 61 years of age and older. Transplantation 2006;81:862-5.

34. McShane PJ, Garrity ER Jr. Impact of the lung allocation score. Semin Respir Crit Care Med 2013;34:275-80.

35. Tong A, Howard K, Jan S, et al. Community preferences for the allocation of solid organs for transplantation: a systematic review. Transplantation 2010;89:796-805. 